\title{
Performance Analyses of Regular Networks with limited number of wavelength converters by employing deflection Routing
}

\author{
Chong Gee Koa ${ }^{2}$, Gerardo Castañón ${ }^{1}$, Alberto Bononi ${ }^{3}$, Su Fong Chien ${ }^{2}$, Andy Lock Yen Low ${ }^{2}$ \\ ${ }^{1}$ Centro de Electrónica y Telecomunicaciones, \\ Instituto Tecnológico y de Estudios Superiores de Monterrey, Ave. \\ Garza Sada 2501, Monterrey NL, México. \\ ${ }^{2}$ Faculty of Engineering \& Technology \\ Multimedia University, Jalan Ayer Keroh Lama, 75450 Melaka, Malaysia. \\ ${ }^{3}$ Dipartamento di Ingegneria dell'Informazione \\ Universita di Parma, I-43100 Parma Italy \\ sfchien@mmu.edu.my \\ Tel: +606-252-3593, Fax: +606-231-6552
}

\begin{abstract}
Optical node performance analysis in terms of number of wavelength converters for a multi-hop regular optical networks under deflection routing is presented in this paper. Based on the computed results for a given number of wavelengths, it is found that in order to achieve the minimum deflection probability at full load, the number of wavelength converters required are at most $60 \%$ of the number of wavelengths. Any additional wavelength converters would not be necessary in reducing the overall deflection probability. These upper bound findings are indeed helpful for network engineers designing a cost effective network node.
\end{abstract}

Keywords- Deflection-routing, Multihop optical networks, wavelength converters, WDM and deflection probability

\section{INTRODUCTION}

The multi-hop optical network based on wavelength division multiplexing (WDM) technology has received extensive study since 1987 [1-2]. Various types of multi-hop regular network topologies have been investigated, two common topologies that are still being used for performance analysis are ShuffleNet and Manhattan Street networks [3]. A simple deflection routing scheme so-called hot potato routing has been proposed to reduce the packet contention probability. This scheme allows packets to be deflected to other nodes when a contention occurs between packets at a particular node. Consequently, deflected packets need to take more hops to reach their destinations. The performance of hot-potato routing scheme based on ShuffleNet topologies has also been studied and compared to the conventional store-and-forward routing scheme [4]. Furthermore, Forghieri et al. have done and presented an extensive study by comparing the hot-potato routing networks to single buffer routing networks [5]. Results show that 2 single buffer deflection routing recovers more than $60 \%$ of the lost throughput of hot-potato with respect to store-and-forward when uniform traffic is assumed. Bononi and Prucnal have investigated various access techniques, to improve the performance of multi-hop deflection routing optical networks. Results show that by-pass queuing access technique outperforms others [6]. Following that, Bononi et al. have extended that work to wavelength convertible multi-hop optical networks with deflection routing. Three new access schemes were proposed and analyzed in [7]. Computed results indicate that with a small number of wavelengths, when transmission is feasible, it may be preferable to use optical buffers rather than employing wavelength converters. Based on the model mentioned previously, Chien et al. evaluated the bit error rate performance in both with/without wavelength conversion multi-hop optical networks by employing convolutional coding [8-9]. Also, Chien et al. proposed hotpotato routing with buffers to enhance the throughput/delay performance and reduce the number of wavelength converters needed, compared to the hot-potato routing multi-hop wavelength convertible networks [10].

In this paper, we further investigate the performance of multihop wavelength convertible ShuffleNet with hot-potato routing which employs limited number of wavelength converters. The probability model presented in [7] is extended in order to have a more accurate analytical model. All formulations are addressed in section 3 . We consider the main contribution of this paper to be as important as the work previously presented, such as the issues of sparse wavelength conversion and wavelength converters placement in optical networks [11-12]. Based on this research, we expect that the number of wavelength converters needed to achieve the highest throughput in a multi-hop ShuffleNet for each node

This project is supported by the Malaysia Ministry of

Science, Technology \& Innovation - Intensification of

Research in Priority Areas (IRPA) 09-99-01-0070-EA066 
can be optimized. The characteristics of multi-hop ShuffleNet with limited number of converters in each

node can be further explored.

\section{DESCRIPTION OF LOGICAL NODE OPERATION}

Fig. 1 illustrates the logical structure of a node. There are two input and output fibers in each node. All of the number of wavelengths $n_{w}$ from each input fiber are de-multiplexed and sent to a stack of $n_{w}$ modules. It is assumed that all functions in each module, such as packets absorption, injection, wavelength conversion ( $\lambda$-conversion) and routing are sequentially and independently performed. In the final stage, packets are re-multiplexed onto the output fiber to be sent to the next nodes according to the shortest path algorithm. The absorption block is assumed to have one receiver per input wavelength to ensure that all packets that are destined to the node can be removed. On the other hand, the injection block transmits the locally generated packets and the process can only take place when there is at least one empty slot. The function of wavelength conversion block is to solve packet contentions by rearranging the packets on the various wavelengths to minimize the wavelength conflicts before sending to the routing block. There are all together $n_{c}$ wavelength converters in the wavelength conversion block, where $n_{c}$ is in the range of $1 \leq n_{c} \leq n_{w}$. The routing is a simple un-buffered $2 \times 2$ switch. In case of output contention, packets are selected randomly, and selected packets will be deflected to the undesired port. Also, in this paper we assume uniform traffic, which allows simple comparisons of node structures and control algorithms, and the conclusions usually hold true in most non-pathological non-uniform traffic scenarios [5].

It is noted that four categories of packets are defined. "Don't Care" (DC) is a packet that can take either output whereas "Care 1" $(\mathrm{C} 1)$ is a packet that cares to exit on output 1. Similarly, "Care 2" is a packet that cares to exit on output 2. "For Node" (FN) means a packet is destined to a node. Slots on each wavelength can be empty (E), or carry a FN packet, or carry a $\mathrm{C} 1$ packet, or carry a $\mathrm{C} 2$ packet, or carry a DC packet.

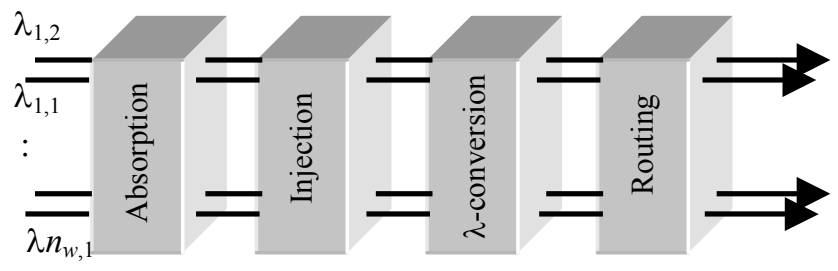

Fig.1 Logical structure of node structure.

\section{WAVELENGTH CONVERSION ALGORITHM AND TRAFFIC ANALYSIS}

Before a packet is sent to the routing block, it is directed to the wavelength conversion block to solve contention and to avoid deflections. The wavelength conversion algorithm is as follows:
Modules with contending input packets are grouped in two sets: set $A(C 1, C 1)$ and set $B(C 2, C 2)$. Let a be the number of elements in set $\mathrm{A}$ and let $\mathrm{b}$ be the number of elements in Set B. Subsequently, modules without input contention are grouped in other three sets: 1) set $\mathrm{C}$, modules that do not contain any $\mathrm{C} 1$ packets and empty slots ((C2,DC),(DC,C2), $(\mathrm{DC}, \mathrm{DC}))$; 2) set $\mathrm{F}$, modules that do not contain any $\mathrm{C} 1$ packets and contain at least one empty slot ((C2,E), (E,C2), (DC,E), (E,DC), (E,E)); 3) and set $\mathrm{G}$, modules that contain a $\mathrm{C} 1$ packet $((\mathrm{C} 1, \mathrm{E}),(\mathrm{E}, \mathrm{C} 1),(\mathrm{C} 1, \mathrm{DC}),(\mathrm{DC}, \mathrm{C} 1),(\mathrm{C} 1, \mathrm{C} 2)$, $(\mathrm{C} 2, \mathrm{C} 1))$. Let $\mathrm{c}$ be the number of elements in $\mathrm{C}$ and let $\mathrm{f}$ the number of elements in F. In the following algorithm we will assume that $\mathrm{a} \geq \mathrm{b}$ and conversion priority will be given to $\mathrm{C} 1$ packets otherwise if $b \geq a$ reverse the reasoning.

\section{A. Algorithms}

To solve contentions and avoid deflections at the routing block, the node controller uses the following algorithm presented in pseudocode

\section{$/ * \boldsymbol{B E} \boldsymbol{G I N}$ */}

Get $\mathrm{a}, \mathrm{b}, \mathrm{n}_{\mathrm{c}}, \mathrm{f}$, and c variables

$\mathrm{a}_{1}=\mathrm{a} ; \quad \%$ initialize $a_{l}$

$\mathrm{b}_{1}=\mathrm{b} ; \%$ initialize $b_{l}$

$\mathrm{c}_{1}=\mathrm{c} ; \quad \%$ initialize $c_{l}$

$\mathrm{f}_{\mathrm{l}}=\mathrm{f} ; \quad \%$ initialize $f_{l}$

$\mathrm{n}_{\mathrm{cl}}=\mathrm{n}_{\mathrm{c}} ; \%$ initialize $n_{c l}$

While $\left(\mathrm{b}_{1} \geq 1\right) \&\left(\right.$ floor $\left.\left(\mathrm{n}_{\mathrm{cl}} / 2\right) \geq 1\right) \%$ swap packets

between modules $A$ and $B$

\{

from the $a_{1}\left(b_{1}\right)$ modules with conflict in $A(B)$ select at random one packet from each set and swap them, this will remove contentions in modules of both sets $\mathrm{A}$ and $\mathrm{B}$; $\%$ Swapping is achieved by interchanging the packets and also the wavelength of the two packets. $\mathrm{b}_{1}=\mathrm{b}_{1}-1 ; \mathrm{a}_{1}=\mathrm{a}_{1}-1 ; \mathrm{n}_{\mathrm{cl}}=\mathrm{n}_{\mathrm{cl}}-2 ; \quad \%$ update variables \}

while $\left(\mathrm{a}_{1} \geq 1\right) \&\left(\mathrm{f}_{1} \geq 1\right) \&\left(\mathrm{n}_{\mathrm{cl}} \geq 1\right)$

$\%$ continue with the translation process from $A$ to $F$

from the remaining $\mathrm{a}_{1}$ modules with conflict in A select at random one packet and translate it to the empty slot of one of the available $\mathrm{f}_{1}$ modules in $\mathrm{F}$;

\}

$\mathrm{f}_{1}=\mathrm{f}_{1}-1 ; \mathrm{a}_{1}=\mathrm{a}_{1}-1 ; \mathrm{n}_{\mathrm{cl}}=\mathrm{n}_{\mathrm{cl}}-1 ; \%$ update variables

while $\left(\mathrm{a}_{1} \geq 1\right) \&\left(\mathrm{c}_{1} \geq 1\right) \&\left(\right.$ floor $\left.\left(\mathrm{n}_{\mathrm{cl}} / 2\right) \geq 1\right)$

$\%$ swap packets between modules $A$ and $C$

\{

from the remaining $\mathrm{a}_{1}$ modules with conflict in $\mathrm{A}$ and from the available $\mathrm{c}_{1}$ modules in $\mathrm{C}$ select at random one packet from each set and swap them, this will remove contentions in modules of set $\mathrm{A}$;

$\mathrm{c}_{1}=\mathrm{c}_{1}-1 ; \mathrm{a}_{1}=\mathrm{a}_{1}-1 ; \mathrm{n}_{\mathrm{cl}}=\mathrm{n}_{\mathrm{cl}}-2 ; \%$ update variables \}

/ END */ 


\section{$B$.}

Slot utilization

To solve contentions and avoid deflections at the routing block, the node controller uses the following algorithm presented in pseudocode.

Let $u$ be the input slot utilization, i.e., the probability that a slot from the input carries a packet. Define $P_{\mathrm{dc}}$ as the probability that a packet is don't care (DC), i.e., that the packet can take either output, and $r$ be the probability that the packet is destined for the node. Let $g$ be the packet-generating probability and $P_{\mathrm{dc} 0}$ be the probability of DC when a new packet is injected into the network. At every clock cycle the input slots are assumed to be the independent random variables with the same probability distribution $f_{i}=\left\{P\left(i_{j}=s\right)\right.$, $s \in\{E, \mathrm{DC}, \mathrm{C} 2, \mathrm{C} 1\}\}$, where $\mathrm{C} 1(\mathrm{C} 2)$ is the packet that cares to exit on output $1(2), j=1,2, \ldots, 2 n_{w}$. At the moment when packets reach the absorption block, $f_{i}$ can be rewritten as

$$
f_{i}=\left\{f_{i}(E), f_{i}(\mathrm{DC}), f_{i}(C)\right\}=\left\{1-u(1-r), u P_{\mathrm{dc}}, u\left(P_{\mathrm{dc}}-r\right)\right\}
$$

It is assumed that among the care packets, both output 1 and output 2 are equally likely. At steady state and under uniform traffic assumption, at each node and clock time, the average number of absorbed packets per wavelength $S_{\text {abs }}$ must be equal to the average number of injected packets $S_{\mathrm{inj}}$, and hence their common value $T$ can be termed as throughput per node per wavelength $S$. Since there is in average $r u$ packets destined to the arriving node per wavelength per input, and all of them are absorbed, we have $S_{\text {abs }}=r u$. By Little's law, throughput $T$ for two-connected networks is obtained i.e., $2 u / H$, where $H$ is the average number of hops. Following that $r$ can be obtained immediately as $r=1 / H$. According to the access scheme mentioned, the average number of packets injected per wavelength can be written as $S_{\text {inj }}=g\left\{1-\left[1 .-f_{i}(E)\right]^{2}\right\}$ and the closed expression for $u$ is [7]:

$$
u=\frac{\sqrt{r^{2}+g^{2}(1-r)^{2}}-r}{g(1-r)^{2}}
$$

\section{Deflection Probability}

Due to the properties of the regular network topology and uniform traffic assumptions, the deflection probability $\mathrm{d}$ of a care test packet (TP) at an intermediate node, and deflection probability $\mathrm{d}_{0}$ of a care TP at its injection block can be derived. A deflection happens on TP if TP enters the conversion block in a module with another competing packet and the contention is not removed in the conversion block. Let $\mathrm{P}_{\text {cont }}$ be the probability that TP belongs to a particular module in which contention is not solved and $\mathrm{P}_{\mathrm{c}}$ is the combination of contention probabilities of all modules, according to the wavelength conversion algorithm mentioned previously, both $\mathrm{P}_{\text {cont }}$ and $\mathrm{P}_{\mathrm{c}}$ could be derived as:

$$
P_{c}=\sum_{s} P_{c o n t} \cdot P(a, b, c, f)
$$

where

$$
\begin{aligned}
& S \in:\{(a, b, c, f): \\
& \left.\qquad n_{w} \geq(a+b+c+f) \geq 1 ; a>0 ; b \geq 0 ; c \geq 0 ; f \geq 0\right\}
\end{aligned}
$$

is the set of feasible states of the four variables $(\mathrm{a}, \mathrm{b}, \mathrm{c}, \mathrm{f})$ where contentions remain for the test packet (TP). For programming purposes $S$ can be found as follows. Fix $1 \leq \mathrm{a} \leq \mathrm{n}_{\mathrm{w}}$ (it must be larger than 0 since the TP is in A). Then select the number of modules in $\mathrm{B}: 0 \leq \mathrm{b} \leq \mathrm{n}_{\mathrm{w}}-\mathrm{a}$. Also select the number of modules in $\mathrm{F}: 0 \leq \mathrm{f} \leq \mathrm{n}_{\mathrm{w}}-\mathrm{a}-\mathrm{b}$. Finally we select the number of modules in $\mathrm{C}$ : $0 \leq \mathrm{c} \leq \mathrm{n}_{\mathrm{w}}-\mathrm{a}-\mathrm{b}-\mathrm{f}$. This guarantees that $\mathrm{a}+\mathrm{b}+\mathrm{f}+\mathrm{c} \leq \mathrm{n}_{\mathrm{w}}$. Now to obtain the probability $\mathrm{P}_{\text {cont }}$ we can use the following equation:

$$
P_{\text {cont }}=\frac{a_{1}}{a}
$$

where $\mathrm{a}$ is the number of contentions in set $\mathrm{A}$ and $\mathrm{a}_{1}$ is the number of remaining contentions. Therefore since the selection of packets was done randomly, the probability that the test packet TP is in contention is $P_{\text {cont }}=a_{1} / a$. The parameter $a_{1}$ can be computed using the algorithm already mentioned in subsection 3.1.

Now $\mathrm{P}(\mathrm{a}, \mathrm{b}, \mathrm{c}, \mathrm{f})$ is derived using the following equation which is the probability that the four variables $(\mathrm{a}, \mathrm{b}, \mathrm{c}, \mathrm{f})$ may occur

$$
\begin{aligned}
& P(a, b, c, f)=P(T P) \times \\
& {\left[\frac{\left(n_{w}-1\right) ! P(A)^{(a-1)} P(B)^{b} P(C)^{c} P(F)^{f} P(G)^{\left(n_{w}-a-b-c-f\right)}}{(a-1) ! b ! c ! f !\left(n_{w}-a-b-c-f\right) !}\right]}
\end{aligned}
$$

Note that the events TP, A, B, C, F and G are defined as follows:

$T P=\{$ the packet conflicting with flow-through test packet TP is $\mathrm{C} 1\}$,

$A=\{$ a sub-module with a conflict $(\mathrm{C} 1, \mathrm{C} 1)\}$,

$B=\{$ a sub-module with a conflict $(\mathrm{C} 2, \mathrm{C} 2)\}$,

$C=\{$ a sub-module without conflicts nor $\mathrm{C} 1 \mathrm{~s}$ nor $\mathrm{E}\}$,

$F=\{$ a sub-module without conflicts nor $\mathrm{C} 1 \mathrm{~s}$ with at least one

$\mathrm{E}\}$,

$G=\{$ a sub-module with 1 and only one $\mathrm{C} 1\}$.

The probability for each case can be written as

$$
\begin{aligned}
& P(T P)=\frac{u\left(1-P_{d c}-r\right)+g(1-u+u r)\left(1-P_{d c 0}\right)}{2} \\
& P(A)=P(B)=\left[\frac{u\left(1-P_{d c}-r\right)}{2}\right]^{2} \\
& +\left[\frac{u\left(1-P_{d c}-r\right)}{2}\right](1-u+u r) g\left(\frac{1-P_{d c 0}}{2}\right)
\end{aligned}
$$




$$
\begin{aligned}
& P(C)=\left\{\left(1-(1-u+u r)-\frac{u\left(1-P_{d c}-r\right)}{2}\right)^{2}-\left(\frac{u\left(1-P_{d c}-r\right)}{2}\right)^{2}\right\} \\
& +\left[2\left(1-(1-u+u r)-\frac{u\left(1-P_{d c}-r\right)}{2}\right)(1-u+u r)\right] \\
& \times\left[1-\left(\frac{g\left(1-P_{d c 0}\right)}{2}\right)\right] \\
& P(F)=(1-u+u r)^{2}\left[1-\left(\frac{g\left(1-P_{d c 0}\right)}{2}\right)\right] \\
& P(G)=1-P(A)-P(B)-P(C)-P(F)
\end{aligned}
$$

Hence, the deflection probability of $T P$ after the conversion block can be written as

$$
d=P_{c} / 2
$$

The initial deflection probability of a $T P$ at the injection, $d_{0}$ is identical to that of $d$, except $P\{T P\}$ have to change to

$$
P(T P)=u\left(1-P_{d c}-r\right) / 2
$$

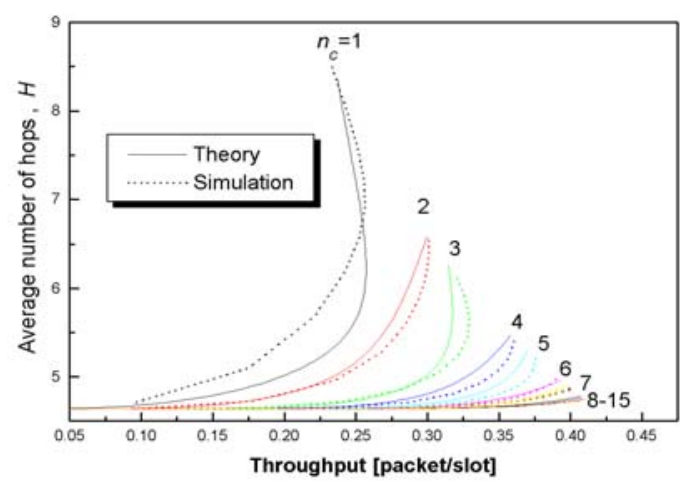

Fig.2(a) Average number of Hops $H v s$. Throughput per wavelength $T$ for case $n_{w}=15$ wavelengths in SFNet-64.

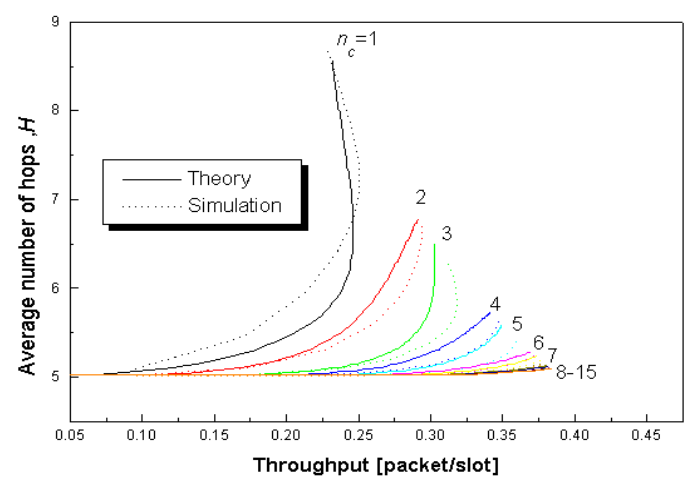

Fig.2(b) Average number of Hops $H$ vs. Throughput per wavelength $T$ for case $n_{w}=15$ wavelengths in MSN-64.

\section{RESULTS AND DISCUSSION}

This paper studies the deflection probability at care nodes with reference to the link utilization in the analysis of 64-node ShuffleNet. A simulation was carried out to validate the accuracy of the analytical model. Both, simulation and theoretical result of average number of hops in the 64-node ShuffleNet and 64-node Manhanttan Street for $n_{w}=15$ against network throughput have been examined as shown in Fig. 2(a) and Fig. 2(b). The discrepancies in result between theory and simulation are mostly due to traffic inhomogeneities for the case of ShuffleNets [7]. Although the network is regular and the traffic is uniform, the number of $\mathrm{C} 1$ and $\mathrm{C} 2$ packets received from the two input links of a module will be imbalanced. However, the discrepancies are reasonability low for $n_{c}<6$ and negligible for $n_{c} \geq 6$. Furthermore, an interesting bi-stable characteristic is found to happen when $n_{c}=1$ and $n_{c}$ $=3$ for both simulation and theoretical result. This bi-stable characteristic is due to the incapacity of the system to solve packet contentions (because there are not enough converters) and traffic imbalance. In the case of $n_{c}=1$, only one $(\mathrm{C} 1, \mathrm{C} 1)$ contention can be solved when $f>0$ and all the remaining $(\mathrm{C} 1, \mathrm{C} 1)$ and $(\mathrm{C} 2, \mathrm{C} 2)$ contentions can not be solved creating additional traffic imbalance in the network. Also, there is a point in the throughput when the use of the converter saturates and the network operates as if wavelengths were independent i.e. no converters due to this deflections increase and therefore average number of hops increases and throughput decreases. Also, in Fig. 2(a) and Fig. 2(b), it is found that the average number of hops decreases with the increment of the number of converters. However, it will reach a level whereby any additional increment in the number of wavelength converters will have insignificant improvement of the average number of hops required.

Figure 3(a) shows the deflection probability at full load versus the ratio of number of converters to the number of wavelengths $\left(n_{c} / n_{w}\right)$ for both 64-Node and 324-Node ShuffleNet. It is found that the deflection probability at full load will reach a saturation level when the number of converters in each node is approximately $60 \%$ of the number of wavelengths in the networks and is independent of the network size. It is also evident that when $n_{w}$ becomes larger (e.g. 30) the deflection probability for the ratio $n_{c} / n_{w}>0.6$ has no difference, again, deflection probability is independent of network size in this circumstance. It is noted that the deflection probability for smaller network size is greater than the larger network size because the ratio of traffic per node is greater and this causes packet to be deflected more frequently. Figure 3(b) shows the deflection probability with a value of link utilization that used in practical network designing, that is $u=0.5$. Compare to Fig. 3(a), Fig. 3(b) gives a smoother curve for $n_{w}=10$ and 20. The optimum ratio of $n_{c} / n_{w}$ when $u=0.5$ also has a smaller value, that is, 0.52 compare to the optimum ratio of $n_{c} / n_{w}$ when $u=1.0$.

The same characteristic is found that happen in Manhantan Street Network as well, and the result is shown in Fig. 3(c) and Fig. 3(d). 


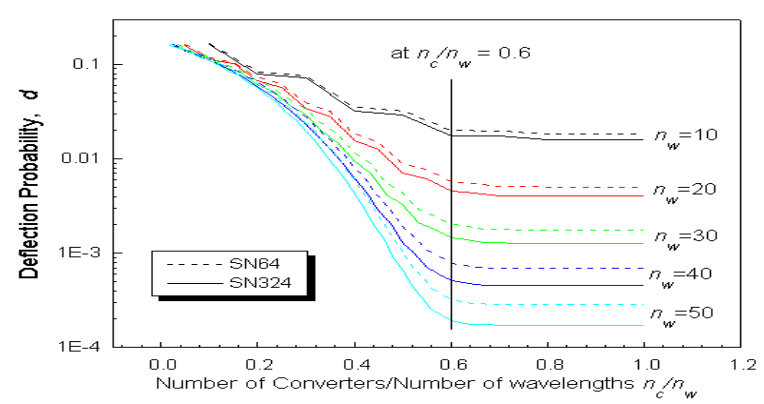

Fig. 3(a) Deflection probability $d v s$. Number of Converters/Number of Wavelengths $n_{c} / n_{w}$ when $u=1.0$ for SFNet-64 and SN324

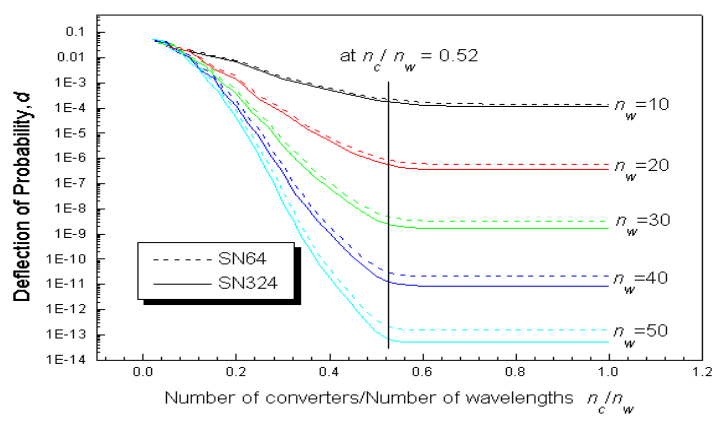

Fig. 3(b) Deflection probability $d v s$. Number of Converters/Number of Wavelengths $n_{c} / n_{w}$ when $u=0.5$ for SFNet-64 and SN324.

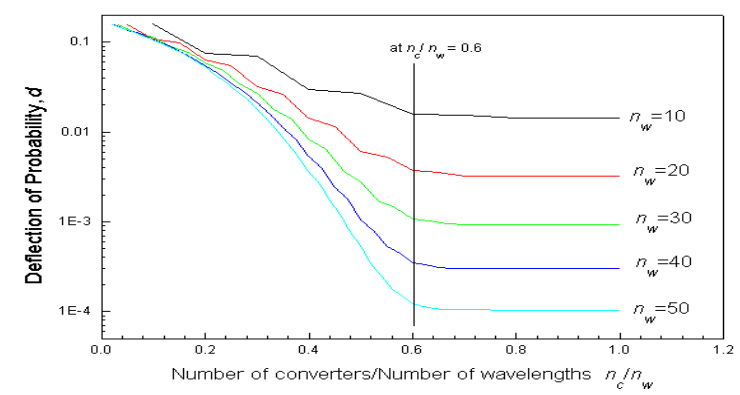

Fig. 3(c) Deflection probability $d v s$. Number of Converters/Number of Wavelengths $n_{c} / n_{w}$ when $u=1.0$ for MS64.

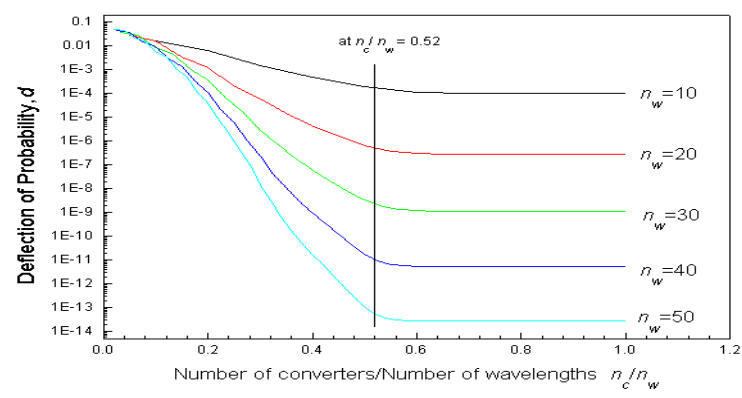

Fig. 3(d) Deflection probability $d v s$. Number of Converters/Number of Wavelengths $n_{c} / n_{w}$ when $u=0.5$ for MS64.

\section{CONCLUSION}

We have performed a detail analysis of multi-hop wavelength convertible ShuffleNet with limited number of wavelength converters employing deflection routing. Based on our study, the required number of wavelength converters is only $60 \%$ or less of the number of optical carriers (wavelengths) in each node in order to reduce the propagation delay in a full loaded optical network. Any additional wavelength converters will not be useful and necessary in reducing the overall network deflection probability to obtain the maximum throughput per wavelength. Thus, from this analysis, the network cost can be optimized in terms of number of wavelengths usage and wavelengths converters utilization.

\section{REFERENCES}

[1] A. S. Acampora and A. Shah, "A multichannel multihop local lightwave network," GLOBECOM'87, Conf. Rec., pp. 1459-1467, Nov. 1987.

[2] B. Ramamurthy, D. Datta, H. Feng, J. P. Heritage, and B. Mukherjee, "Transparent vs. opaque vs. translucent wavelength-routed optical networks," in Optical Fiber Communication Conference (OFC 1999) (Optical Society of America, Washington, D.C., 1999), Vol. 1, pp. 59-61.

[3] I. Chlamtac, A. Farago, and T. Zhang, "Lightpath (wavelength) routing in large WDM networks," IEEE J. Sel. Areas Commun. 14, 909-913 (1996).

[4] A. S. Acampora and S.I.A. Shah, "Multihop lightwave networks: A comparison of store-and-forward and hotpotato routing," INFOCOM'91 Conf. Proc., pp. 10-19, April 1991.

[5] F. Forghieri, A. Bononi, and P.R. Prucnal, "Analysis and comparison of hot-potato and single-buffer deflection routing in very high bit rate optical mesh networks," IEEE Trans. Commun. 43, 88-89 (1995).

[6] A. Bononi, and P.R. Prucnal, " Analysis Evaluation of Improved Access Techniques in Deflection Routing Networks," IEEE/ACM Trans. Netw 4, 726-730 (1996).

[7] A. Bononi, Gerardo A. Castanon and Ozan K. Tonguz, "Analysis of Hot-Potato Optical Networks with Wavelength Conversion," J. Lightwave Technol. 17, 525534 (1999).

[8] S.F. Chien, K. Takahashi and S.P. Majumder, "Multihop optical network with Convolutional coding," J. Opt. Netw 1, 66-73 (2002), http://www.osajon.org/abstract.cfm?URI=JON-1-1-66.

[9] S.F. Chien, K. Takahashi, and S. P. Majumder, "Performance of wavelength-convertible multihop optical ShuffleNets employing convolutional coding under hotpotato routing," J. Opt. Netw. 1, 93-101 (2002), http://www.osa-jon.org/abstract.cfm?URI=JON-1-2-94.

[10] S.F. Chien, K. Takahashi, S.P. Marjumder, "Performance of Wavelength Convertible Multi-hop Optical Network under Deflection Routing with a Delay-line Optical Buffer," in The 4th International Conference on Transparent Optical Networks (ICTON 2002), Mo.B.5, pp. 46-48 April, Poland.

[11] M. A. Subramaniam and A. K. Somani, "All optical networks with sparse wavelength conversion", IEEE/ACM Trans. Netw., 4, 544-557 (1996).

[12] M. A. Subramaniam and A. K. Somani, "On optimal converter placement in wavelength routed networks", IEEE/ACM Trans. Netw., 7, 754-767(1999). 